\title{
Compact Focusing Grating Couplers Between Optical Fibers and Silicon-on-Insulator Photonic Wire Waveguides
}

\author{
F. Van Laere, W. Bogaerts, D. Taillaert, P. Dumon, D. Van Thourhout and R. Baets \\ Department of Information Technology (INTEC), Ghent University-IMEC, Sint-Pietersnieuwstraat 41, 9000, Gent, Belgium \\ frederik.vanlaere@intec.UGent.be
}

\begin{abstract}
We present compact and broadband grating couplers in Silicon-on-Insulator, between single-mode fiber and nanophotonic waveguides. Curved gratings allow for a very short transition between wide waveguides and photonic wires, resulting in very compact devices.

(C)2006 Optical Society of America

OCIS codes: (230.3120) Integrated optics Devices; (230.1950) Diffraction gratings; (250.5300) Photonic integrated circuits; (230.7370) Waveguides;
\end{abstract}

\section{Introduction}

Using high refractive index contrast materials is the route towards ultra-dense photonic integrated circuits. The last few years, a lot of very compact components were demonstrated in Silicon-on-Insulator (SOI) [1]. For this highindex contrast material system, CMOS-fabrication processes can be used, allowing for volume fabrication. An important problem remains the interface with the outside world. The large difference in mode-size between a singlemode optical fiber and nanophotonic on-chip waveguides results in large coupling losses and high packaging costs.

Several solutions have been proposed. A low-loss, broadband edge-coupling method using an inverted taper is described in [2], [3], but this approach requires lensed or special fibers with high numerical aperture. We use compact diffraction gratings for near-vertical coupling between standard single-mode fiber and nanophotonic waveguides. This obviates the need for a cleaved facet and light can be coupled in and out everywhere on the chip, allowing for wafer-scale testing of components. The principle is shown in Fig. 1. Due to the high vertical refractive index contrast of SOI, the gratings are strong, resulting in compact and broadband devices. A coupling efficiency of $33 \%$ for a $10 \times 10 \mu \mathrm{m}^{2}$ grating has been demonstrated in [4]. Other properties of the coupler are its $1 \mathrm{~dB}$ bandwidth of around $40 \mathrm{~nm}$ and good alignment tolerances $(+/-2 \mu \mathrm{m}$ for $1 \mathrm{~dB}$ excess loss). By adding a bottom mirror, the coupling efficiency is increased to a measured efficiency of 69\% [5]. A 2-D grating can be used as a coupler with integrated polarization splitter, allowing a polarization diversity circuit [6].

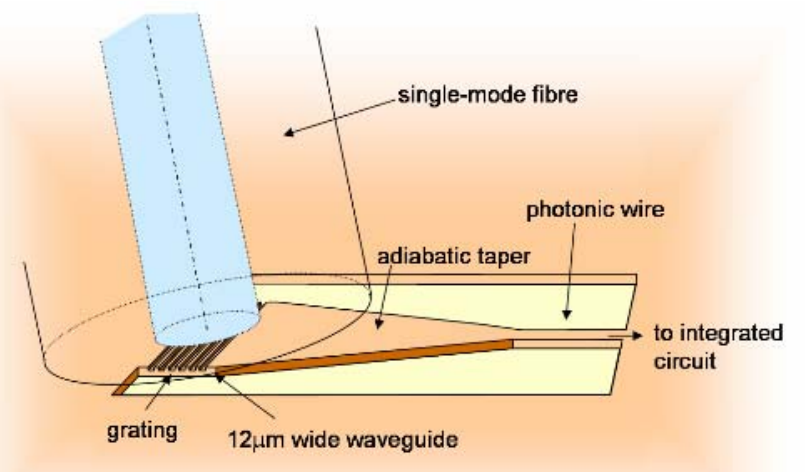

Fig. 1. Principle of a grating coupler for coupling between SM-fiber and nanophotonic waveguides.

\section{Focusing grating coupler}

When using a straight 1-D grating (Fig. 1) the light from the fiber is first diffracted into a 10-12 $\mu \mathrm{m}$ wide, multimode waveguide, which is then adiabatically tapered down to the photonic wire $(500 \mathrm{~nm}$ wide). As a result, we have a very compact grating in combination with a relatively long taper (a parabolic taper needs to be at least $100 \mu \mathrm{m}$ long). However, when curving the grating lines, the diffracted light can be directly focused into the photonic wire aperture over a much shorter distance. The use of focusing gratings has been studied for use in integrated-optic disk pickup devices [7], but also for coupling to waveguides [8]. However, in these cases weak (long) gratings were being used, with focal distances in the order of $\mathrm{mm}$. In [9], the use of focusing grating couplers for coupling to SOI waveguides was briefly mentioned, but no extensive results were reported as yet. 
In this paper, we demonstrate coupling structures based on focusing gratings, with at least an 8-fold length reduction without performance penalty. This results in a coupling structure between fiber and photonic wire with a footprint of $18.5 \mu \mathrm{m} \times 28 \mu \mathrm{m}$.

\section{Design method}

While rigorous design of the focusing grating couplers would require full 3-D simulations, we used an approximation starting from the standard linear grating couplers, which were designed in 2-D. Near-vertical coupling at 10 degrees is used to avoid second-order reflection. These couplers work for TE-polarization (electric field parallel to the grating lines) and have a period of $630 \mathrm{~nm}$, a 50\% filling factor and an etch depth of $70 \mathrm{~nm}$. The coupling efficiency is $37 \%$ [10].

Subsequently, the focusing is implemented by curving the grating lines. The curvature can be calculated from the phase difference between the input wave (from the fiber) and the output wave (focusing wave towards the photonic wire). We have applied the formulas elaborated in [8]:

$$
q \lambda=z n_{\text {air }} \cos \theta-n_{\text {eff }} \sqrt{y^{2}+z^{2}}
$$

with $z$ the coordinate in the propagation direction, $y$ the coordinate in the lateral direction, $q$ the grating line number and $\theta$ the coupling angle. The required effective index $n_{\text {eff }}$ in the grating area is taken from the straight grating (CAMFR simulation). We have ignored the fact that the effective index in the grating area differs from the effective index in the slab. For vertical coupling $\left(\theta=90^{\circ}\right)$, the grating lines become concentric circles focusing on the common center. When the fiber is tilted (e.g. $\theta=80^{\circ}$ ), the grating lines describe ellipses with one common focal point.

\section{Fabrication}

The SOI-structures were fabricated using standard CMOS tools, using $248 \mathrm{~nm}$ deep UV lithography for pattern definition[1]. The gratings were etched $70 \mathrm{~nm}$ into the top silicon layer. The waveguides and tapers were defined in a separate patterning step, which is etched completely through the $220 \mathrm{~nm}$ silicon core. Alignment between grating and waveguides is of the order of $50 \mathrm{~nm}$. SEM-micrographs of fabricated structures are shown in Fig. 2.
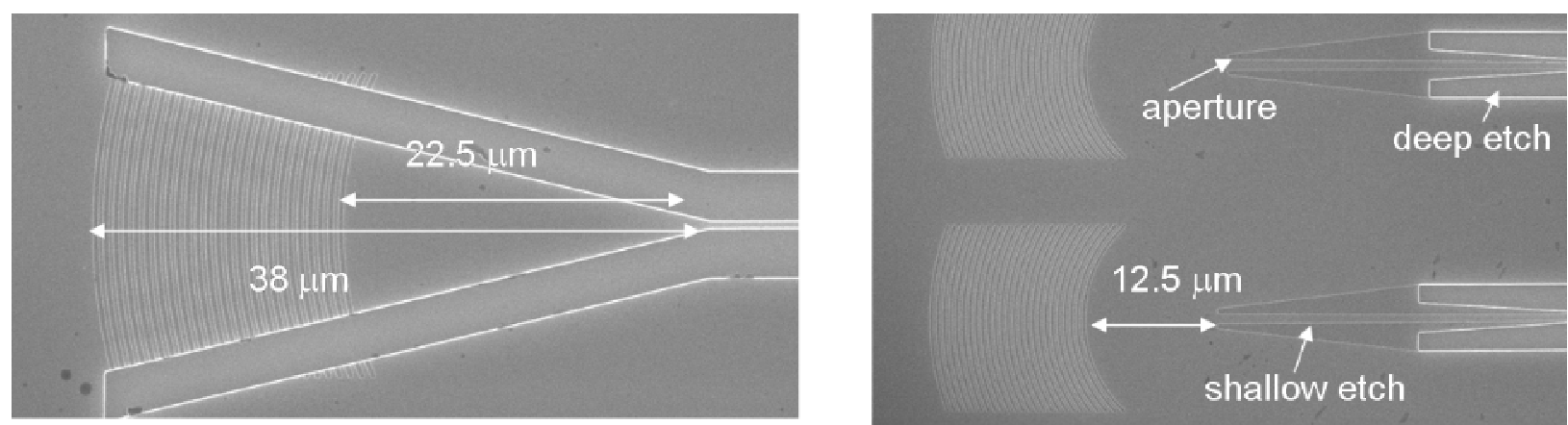

Fig. 2. SEM- micrograph of fabricated structures. Left: Focusing grating in a short linear taper configuration (38 $\mu \mathrm{m}$ total length in this case, shortest fabricated is $28 \mu \mathrm{m}$ ) . Right: Focusing grating without taper focusing onto a low-contrast aperture.

\section{Measurements}

We have characterized the coupling efficiency through fiber-to-fiber transmission measurements. A standard singlemode fiber, connected to a Superluminescent-LED $(1500 \mathrm{~nm}<\lambda<1600 \mathrm{~nm})$, is positioned above the input grating at an angle of 10 degrees with respect to the vertical axis. The ASE of the S-LED is used as the input power. Another fiber, connected to a spectrum analyzer, is positioned (also at 10 degrees) above the output grating. Only the input grating is curved, while the output grating is the standard linear grating with a known efficiency. We also included reference structures where the input coupler is also a linear grating. Here, a very long linear taper of 700 micron length is used, for which theoretical transmission is $100 \%$ (shorter tapers could have been used with similar results). The on-chip length between input and output fiber is $3 \mathrm{~mm}$.

From the measured transmission we could directly extract the efficiency of the curved gratings. We have fabricated focusing grating couplers in different configurations, as shown in Fig. 2. The best performance is obtained for focusing grating couplers in combination with a short, linear taper down to a $500 \mathrm{~nm}$ wire (Fig. 2 left) and for couplers without taper, focusing on a low-contrast aperture ranging from $0.8 \mu \mathrm{m}$ to $2 \mu \mathrm{m}$ width (Fig. 2 right). This low-contrast aperture was implemented in a $70 \mathrm{~nm}$ etch (instead of a full etch) using the same two-step etch process used to define the gratings themselves. The transition from low-contrast to high-contrast waveguides is done 
adiabatically over a $30 \mu \mathrm{m}$ length. The fiber-to-fiber loss measurements are shown in Fig. 3. For the short-taper configuration, the performance of the focusing grating coupler is the same as for the reference structure. The fiberto-fiber loss is $10.5 \mathrm{~dB}$, corresponding with a coupling efficiency of a single grating coupler around $30 \%$. The most compact structure of this type has a total length of $28 \mu \mathrm{m}$ (grating+taper). For the shallow-aperture case, the fiber-tofiber loss increases $0.6 \mathrm{~dB}$ for the narrowest $(0.8 \mu \mathrm{m})$ aperture. The coupling efficiency could be further increased by applying a bottom mirror to the grating, as we have demonstrated in [5].

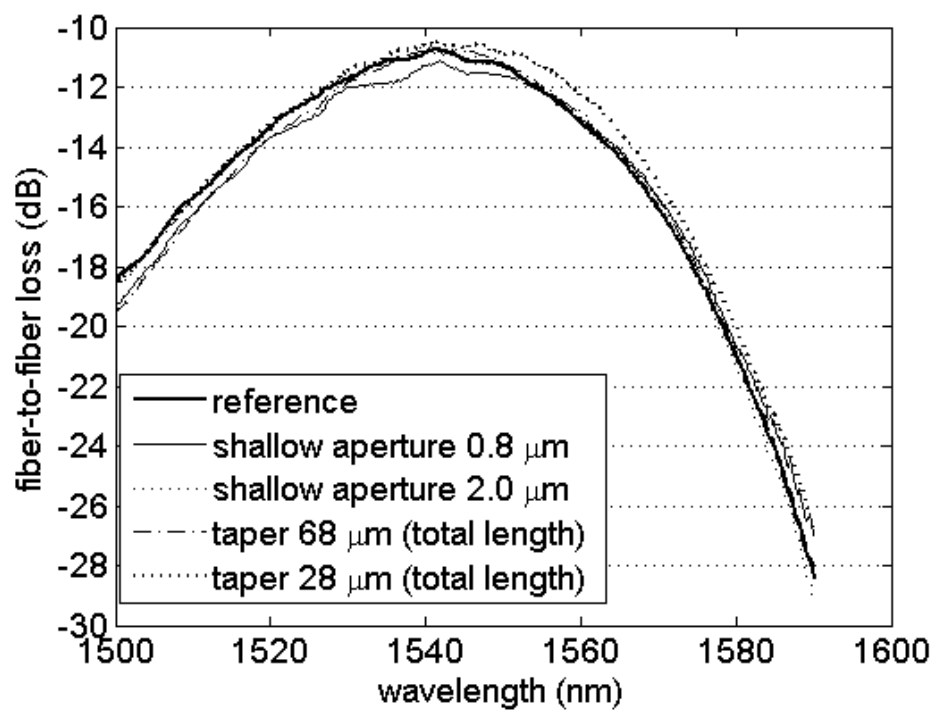

Fig. 3. Measurement results of focusing grating in 2 different configurations: taper and shallow aperture.

\section{Conclusion}

We have demonstrated efficient, broadband and very compact grating couplers, for coupling between single mode fiber and photonic wires in Silicon-on-Insulator. By using focusing (curved) gratings, a long taper between wide waveguide and photonic wire can be avoided. We have compared the performance of different designs experimentally. The best fabricated coupling structure fits into a footprint of $18.5 \mu \mathrm{m}$ (width of the grating) $\times 28 \mu \mathrm{m}$ (length of both grating and linear taper), compared to $12 \mu \mathrm{m}$ (width of the grating) $\times 115.5 \mu \mathrm{m}$ (length grating + shortest possible parabolic taper).

\section{Acknowledgements}

Part of this work was supported by the European Union through the IST-FUNFOX project and the IST-ePIXnet Network of Excellence. Part of this work was supported by the Belgian IAP-PHOTON network project.

\section{References}

[1] W. Bogaerts, R. Baets, P. Dumon, V. Wiaux, S. Beckx, D. Taillaert, B. Luyssaert, J. Van Campenhout, P. Bienstman and D. Van Thourhout, "Nanophotonic waveguides in silicon-on-insulator fabricated with CMOS technology, " IEEE JLT 23, 401-412 (2005)

[2] T. Shoji, T. Tsuchizawa, T. Watanabe, K. Yamada, and H. Morita, "Low loss mode size converter from $0.3 \mu \mathrm{m}$ square Si wire waveguides to singlemode fibres", Electronics Letters 38, 1669-1670 (2002).

[3] G. Roelkens, P. Dumon, W. Bogaerts, D. Van Thourhout, R. Baets, "Efficient Silicon-on-Insulator fiber coupler fabricated using $248 \mathrm{~nm}$ deep UV lithography", IEEE PTL 17, 2613-2615 (2005).

[4] D. Tailaert, R. Baets, P. Dumon, W. Bogaerts, D. Van Thourhout, B. Luyssaert, V. Wiaux, S. Beckx, J. Wouters, "Silicon-on-Insulator Platform for Integrated Wavelength-Selective Components," in Proc. of 2005 IEEE/LEOS Workshop on Fibres and Optical Passive Components (Institute of Electrical and Electronics Engineers, New York, 2005), pp. 115-120.

[5] F. Van Laere, G. Roelkens, J. Schrauwen, D. Taillaert, P. Dumon, W. Bogaerts, D. Van Thourhout, R. Baets, , "Compact grating couplers between optical fibers and Silicon-on-Insulator photonic wire waveguides with 69\% coupling efficiency", OFC2006, PDP15 (2006).

[6] D. Taillaert, H. Chong, P. Borel, L. Frandsen, R. M. De La Rue, R. Baets, "A compact two-dimensional grating coupler used as a polarisation splitter", IEEE PTL 15, 1249-1251 (2003).

[7] S. Ura, T. Suhara, H. Nishihara, J. Koyama, “An integrated-optic disk pickup device”, IEEE JLT LT-4, 913-917 (1986).

[8] R. Waldhäusl, B. Schnabel, P. Dannberg, E-B. Kley, A. Bräuer, W. Karthe, , "Efficient coupling into polymer waveguides by gratings", Applied Optics 36, 9383-9390 (1997).

[9] C. Gunn, "Silicon photonics - Poised to invade local area networks", Photonics Spectra 40 (3), 62-68 (2006)

[10] D. Taillaert, F. Van Laere, M. Ayre, W. Bogaerts, D. Van Thourhout, P. Bienstman, R. Baets, "Grating couplers for coupling between optical fibers and nanophotonic waveguides", Japanese Journal of Applied Physics 45, 6071-6077 (2006). 